\title{
Primera cita de Psalidodon dissensus (Lucena \& Thofehrn 2013) (Characiformes, Characidae) en tributarios de la cuenca del río Uruguay, Misiones, Argentina
}

First record of Psalidodon dissensus (Lucena \& Thofehrn 2013) (Characiformes, Characidae) in tributaries of Uruguay River basin, Misiones, Argentina

Patricia Raquel Araya ${ }^{1}$, Silvia Alicia Flores ${ }^{1 *}$, Marcelo Javier Serrano ${ }^{1}$, Adriana Griselda Barboza ${ }^{1}$

\begin{abstract}
Resumen
Se realiza la primera cita de Psalidodon dissensus en cursos de agua de la provincia de Misiones, a partir de ejemplares provenientes del arroyo Yabotí, afluente del río Uruguay, inmerso en la Reserva de Biosfera Yabotí. Revalidado de la sinonimia del género Astyanax, Psalidodon incluye 21 nuevas combinaciones para la Argentina de la cuales siete están presentes en los sistemas fluviales de Misiones. La ictiofauna de la provincia, perteneciente a la Provincia Parano-Platense, se caracteriza por la elevada diversidad y endemismo que la convierten en territorio de formidable valor biológico y de urgente necesidad de conservación.
\end{abstract}

Palabras claves: diversidad, ictiofauna, Reserva de la Biosfera Yabotí

\begin{abstract}
The first record of Psalidodon dissensus in water courses of the Misiones province, from specimens from the Yabotí stream, a tributary of the Uruguay River, immersed in the Yabotí Biosphere Reserve, is made. Psalidodon, revalidated from the synonymy of the genus Astyanax, includes 21 new combinations for Argentina, of which seven are present in the river systems of Misiones. The ichthyofauna of the province, belonging to the Parano-Platense Province, is characterized by its high diversity and endemism, making it a territory of formidable biological value and in urgent need of conservation.
\end{abstract}

Keywords: Diversity, ichthyofauna, Yabotí Biosphere Reserve

\section{INTRODUCCIÓN}

La ictiofauna de la provincia de Misiones (Argentina) corresponde a la Subregión Neotropical, Provincia de los Grandes Ríos, Clado I (López et al., 2008), Provincia Parano-Platense de Ringuelet (1975), que incluye la mayor diversidad de peces de Argentina: 300 especies continentales de las 570 registradas para Argentina (Mirande y Koerber, 2020). Es un sistema ecológicamente complejo y vulnerable a la acción antrópica, identificado como uno de los 25 biodiversity hotspots (Mittermeier et al., 1998; Myers et al., 2000).

En Misiones se registran 68 áreas protegidas (Ministerio de Ecología y Recursos Naturales Renovables Misiones, 2020), entre ellas la Reserva de la Biosfera Yabotí (RBY). Esta reserva se destaca por una importante red hidrográfica en la que se distingue la cuenca del arroyo Yabotí Guazú, integrada por los

\footnotetext{
1. Facultad de Ciencias Exactas, Químicas y Naturales, Universidad Nacional de Misiones, Argentina.

* Autor para correspondencia: <silvialiciaflores@gmail.com>
} 
arroyos Yabotí Guazú al oeste y el Yabotí Miní al este, que confluyen en un curso único antes de la desembocadura en el río Uruguay. El área ha sido calificada como "Área de Biodiversidad Sobresaliente" (Bertonatti y Corcuera, 2000).

Numerosas especies de la familia Characidae han sido registradas en arroyos de la Reserva de la Biosfera Yabotí, incluyendo especies tradicionalmente incluidas en el género Astyanax (Flores et al., 2015; Flores et al., 2020).

Estudios sobre las relaciones filogenéticas del género, en el contexto de la familia, realizados por Terán et al. (2020) permitieron la recuperación de especies asignadas a otras subfamilias, la asignación de especies a un nuevo género y la categoría de nueva combinación, tal es el caso de Psalidodon dissensus.

El género Psalidodon, fue revalidado de la sinonimia del género Astyanax, que incluye 21 nuevas combinaciones para la Argentina, de la cuales siete están presentes en los sistemas fluviales de misiones, entre ellas Psalidodon dissensus (Lucena y Thofehrn, 2013) (Mirande y Koerber, 2020).

La descripción de $P$. dissensus se realizó con ejemplares del sistema de la laguna los Patos, sistema del río Tramandai y cuenca del río Uruguay (Lucena et al., 2013) y la primera cita para la Argentina proviene de ejemplares del río Uruguay, a la altura de la localidad correntina de Yapeyú (Terán et al., 2016).

El presente trabajo informa la presencia de $P$. dissensus en el arroyo Yabotí, afluentes del río Uruguay, constituyendo la primera cita de la especie en cuencas de la provincia de Misiones.

\section{MATERIALES Y MÉTODOS}

La pesca experimental fue autorizada por Ministerio de Ecología y Recursos Naturales Renovables de la provincia de Misiones, Disposición 008/18 y 036/18. Los ejemplares de referencia fueron depositados en la "Colección Ictiológica, Laboratorio de Genética Evolutiva, Universidad Nacional de Misiones" (LGEP) y en la colección "Peces de la Provincia de Misiones, Laboratorio de Biología, Universidad Nacional de
Misiones" (PPM).

El material examinado provino de la cuenca media del arroyo Yabotí $\left(27^{\circ} 04^{\prime} 08^{\prime \prime} \mathrm{S}\right.$ y $\left.53^{\circ} 53^{\prime} 38^{\prime \prime} \mathrm{W}\right)$, Provincia de Misiones, República Argentina (figura 1). El sector de procedencia, dista $25 \mathrm{~km}$ de la desembocadura en el río Uruguay y 1,6 km aguas abajo de la confluencia de los arroyos Yabotí Guazú y Yabotí Miní. Se alternan extensos y profundos remansos y sectores de rápidos sobre un fondo rocoso basáltico, con escaso depósito de sustrato, principalmente arenoso, en un entorno de abundante vegetación marginal.

El material procedió de las campañas de pesca experimental realizadas en los meses de febrero, mayo, junio y diciembre del año 2018. Las capturas se realizaron con redes de espera monofilamento de malla 2,5 y $3 \mathrm{~cm}$, en tres sectores del arroyo Yabotí; las redes permanecieron caladas durante $24 \mathrm{~h}$ y revisadas cada $12 \mathrm{~h}$. Los ejemplares colectados fueron fijados en formol al $10 \%$ y posteriormente conservados en alcohol $70 \%$.

La merística se realizó utilizando un calibre mecánico decimal, de precisión 0,05 mm y siguiendo la metodología de Fink y Weitzman (1974). Se expresan como porcentaje en la longitud estándar y en la longitud de la cabeza.

\section{RESULTADOS}

\section{Materiales examinados}

Argentina PPM-304 • 1, 77,8 mm; PPM-327 • 1, 75,5 $\mathrm{mm}$; PPM-326 • 1, 79,5 mm; LGEP-829 • 1, 78,2 mm; LGEP-830 • 1, 76,8 mm; LGEP-831 • 1, 75,6 mm; Misiones, Arroyo Yabotí; $27^{\circ} 04^{\prime} 08^{\prime \prime} \mathrm{S}, 53^{\circ} 53^{\prime} 38^{\prime \prime} \mathrm{W}$; 174 m s.n.m.; 10 de junio de 2018; P. Araya, S. Flores, M. Serrano y A. Barboza.

\section{Identificación}

Los ejemplares fueron identificados utilizando la descripción y clave de Lucena et al. (2013) que reportan en $P$. dissensus la siguiente combinación de caracteres: un diente maxilar heptacuspidado, 22-28 radios ramificados en la aleta anal, 35-39 escamas perfora- 
das en la línea lateral, dos manchas humerales, la primera elongada verticalmente, banda lateral conspicua que se intensifica en la región del pedúnculo caudal y continúa en los radios caudales medios, 67 filas de escamas entre el origen de la aleta dorsal y la línea lateral media, la longitud de la cabeza cabe $22,7-27,3 \%$ en la longitud estándar (SL), región predorsal escamada, alto del cuerpo $37,2-44,2 \%$ de SL. Los machos presentan pequeños ganchos óseos en los radios de la aleta anal (figura 2).

Los caracteres morfométricos y merísticos de los ejemplares analizados se presentan en la tabla. La merística de los ejemplares coincide con los rangos hallados por Lucena et al. (2013); sin embargo, el dato promedio en relación con la longitud estándar, es inferior considerando que los ejemplares son de mayor talla.

\section{DISCUSIÓN}

En los últimos años el registro de nuevas especies y cita de ocurrencias en los cursos de agua de la Provincia de Misiones ha permitido la actualización de su ictiofauna. Con esta primera cita de $P$. dissensus, en aguas del territorio misionero, se contabilizan siete especies del género acentuando aún más su carácter de región mega diversa.

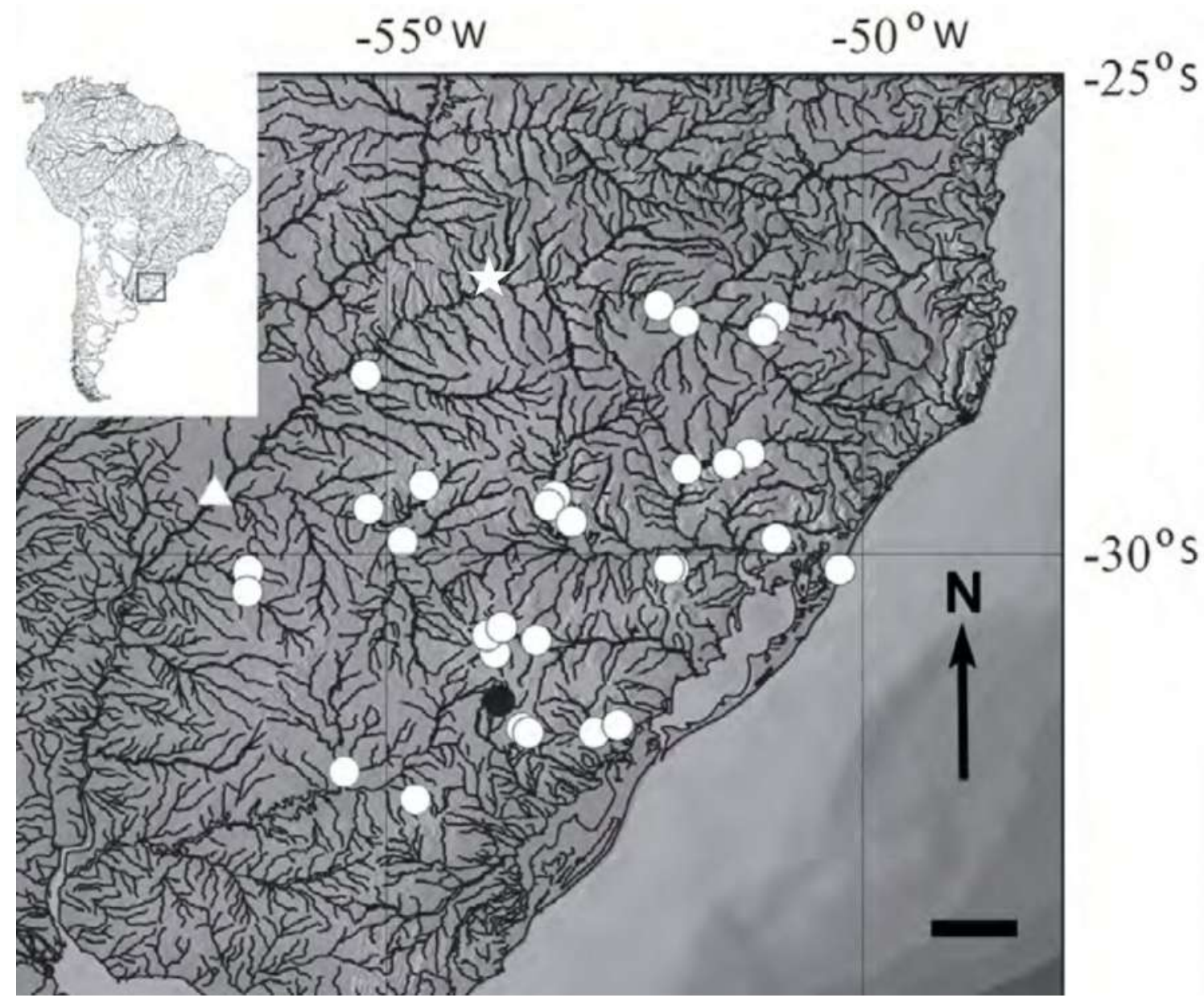

Figura 1. Registros de ocurrencia de Psalidodon disenssus. Mapa modificado de Terán et al. (2016). Círculo negro: localidad tipo. Círculo blanco: ejemplares analizados por Lucena et al. (2013). Triángulo: ejemplares analizados por Terán et al. (2016). Estrella: Registro de la especie en el arroyo Yabotí. Barra de escala = 100 $\mathrm{km}$. 


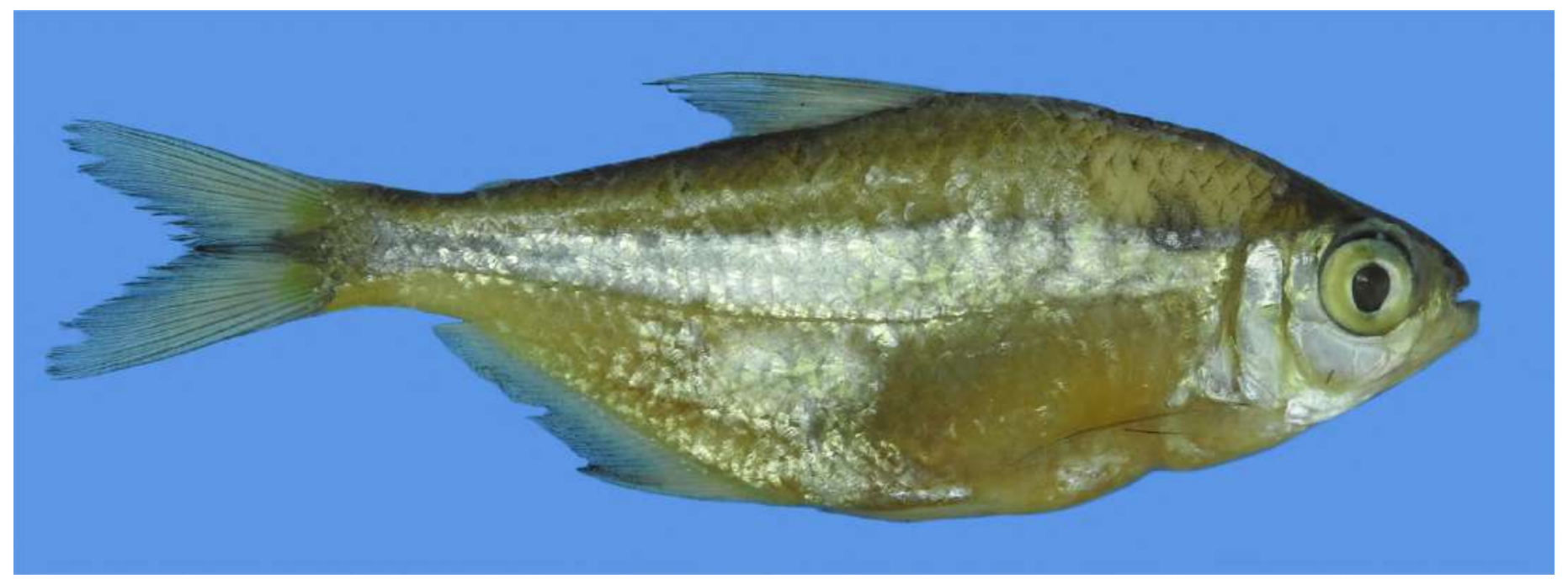

Figura 2. Psalidodon disenssus Lucena \& Thofehrn, 2013.

Tabla. Morfometría de Psalidodon dissensus $(\mathrm{n}=6)$ del arroyo Yabotí, El Soberbio-Misiones

\begin{tabular}{|c|c|c|c|c|c|c|c|c|c|c|}
\hline \multirow{2}{*}{ Ejemplares } & \multirow{2}{*}{$\begin{array}{l}\text { PPM- } \\
304\end{array}$} & \multirow{2}{*}{$\begin{array}{l}\text { PPM- } \\
327\end{array}$} & \multirow{2}{*}{$\begin{array}{l}\text { PPM- } \\
326\end{array}$} & \multirow{2}{*}{$\begin{array}{l}\text { LGEP- } \\
829\end{array}$} & \multirow{2}{*}{$\begin{array}{l}\text { LGEP- } \\
830\end{array}$} & \multirow{2}{*}{$\begin{array}{l}\text { LGEP- } \\
831\end{array}$} & \multirow{2}{*}{ Media } & \multicolumn{2}{|c|}{ Rango } & \multirow{2}{*}{ DE } \\
\hline & & & & & & & & Mín. & Máx. & \\
\hline Longitud estándar $(\mathrm{mm})$ & 77,8 & 75,5 & 79,5 & 78,2 & 76,8 & 75,6 & 77,23 & 75,6 & 79,5 & 1,57 \\
\hline Longitud total (mm) & 98 & 99 & 100 & 103 & 98 & 96 & 99,00 & 96 & 103 & 2,37 \\
\hline Número de radios de aleta anal & 27 & 28 & 28 & 27 & 28 & 28 & 27,67 & 27 & 28 & 0,52 \\
\hline Número de escamas en la línea lateral & 36 & 36 & 35 & 35 & 35 & 36 & 35,50 & 35 & 36 & 0,55 \\
\hline \multicolumn{11}{|l|}{ Porcentaje en la Longitud Estándar } \\
\hline Distancia predorsal & 48,84 & 50,33 & 48,43 & 51,15 & 46,22 & 48,94 & 48,99 & 46,22 & 51,15 & 1,70 \\
\hline Distancia prepélvica & 20,57 & 21,19 & 16,98 & 21,74 & 17,58 & 20,50 & 19,76 & 16,98 & 21,74 & 1,98 \\
\hline Distancia prepectoral & 44,99 & 46,36 & 41,51 & 46,04 & 42,32 & 46,30 & 44,58 & 41,51 & 46,36 & 2,14 \\
\hline Distancia preanal & 24,42 & 25,17 & 24,53 & 24,30 & 24,74 & 25,79 & 24,82 & 24,30 & 25,79 & 0,56 \\
\hline Altura al origen de la aleta dorsal & 50,13 & 64,24 & 59,12 & 63,94 & 59,90 & 64,15 & 60,25 & 50,13 & 64,24 & 5,45 \\
\hline Altura del pendúculo caudal & 34,70 & 37,09 & 32,70 & 35,81 & 36,46 & 37,04 & 35,63 & 32,70 & 37,09 & 1,69 \\
\hline Longitud del pendúculo caudal & 11,57 & 10,60 & 11,32 & 9,59 & 10,42 & 10,58 & 10,68 & 9,59 & 11,57 & 0,70 \\
\hline Longitud de la base de la aleta anal & 12,85 & 13,25 & 12,58 & 12,79 & 13,02 & 13,23 & 12,95 & 12,79 & 13,25 & 0,26 \\
\hline Longitud de la aleta dorsal & 32,13 & 29,14 & 27,67 & 28,13 & 28,65 & 30,42 & 29,36 & 27,67 & 32,13 & 1,66 \\
\hline Longitud de la aleta pélvica & 15,42 & 15,47 & 17,22 & 16,8 & 16,15 & 15,34 & 16,06 & 15,34 & 17,22 & 0,79 \\
\hline Longitud de la aleta pectoral & 20,57 & 20,00 & 22,52 & 21,0 & 21,61 & 21,16 & 21,14 & 20,00 & 22,52 & 0,87 \\
\hline Longitud de la cabeza & 22,36 & 21,76 & 23,84 & 23,1 & 22,40 & 23,54 & 22,84 & 21,76 & 23,84 & 0,80 \\
\hline \multicolumn{11}{|l|}{ Porcentaje en la Longitud de la Cabeza } \\
\hline Longitud del hocico & 19,54 & 27,17 & 22,22 & 21,55 & 21,51 & 23,03 & 22,50 & 19,54 & 27,17 & 2,56 \\
\hline Longitud de la mandíbula superior & 41,95 & 40,46 & 40,56 & 38,67 & 37,79 & 38,76 & 39,70 & 37,79 & 41,95 & 1,55 \\
\hline Longitud del maxilar & 28,74 & 31,79 & 31,67 & 27,62 & 28,49 & 28,09 & 29,40 & 27,62 & 31,79 & 1,84 \\
\hline Diámetro orbital & 41,38 & 42,77 & 42,22 & 41,44 & 41,28 & 40,45 & 41,59 & 42,77 & 40,45 & 0,81 \\
\hline Ancho interorbital & 33,33 & 33,33 & 31,58 & 30,56 & 32,35 & 28,95 & 31,68 & 28,95 & 33,33 & 1,71 \\
\hline
\end{tabular}

DE: desviación estándar 
Finalmente, Misiones es una de las provincias de Argentina con la mayor diversidad íctiofaunistica y elevados endemismos (López et al., 2008), que la tornan en un territorio de formidable valor biológico resaltando la necesidad de conservación de estos sistemas que se presentan altamente vulnerables ante la acción antrópica.

\section{AGRADECIMIENTOS}

Agradecemos la colaboración del Dr. Jorge Casciotta y la Dra. Adriana Almirón, quienes gentilmente participaron en la confirmación de los ejemplares desde la División Zoología Vertebrados, Museo de La Plata (Universidad Nacional de La Plata, Buenos Aires, Argentina).

\section{CONFLICTO DE INTERÉS}

Los autores expresamos que no existen conflictos de intereses de ningún tipo respecto a la publicación del manuscrito que presentamos.

\section{REFERENCIAS}

Almirón, A.E., Azpelicueta, M., Casciotta, J.R., \& López Cazorla, A. (1997). Ichthyogeographic boundary between the Brazilian and Austral subregions in South America, Argentina. Biogeographica, 73(1), 23-30.

Bertonatti, C., \& Corcuera, J. (2000). Situación Ambiental Argentina. Fundación Vida Silvestre. http://guia_guanacos.lumadoc.com.ar/assets/librosituacion-ambiental-argentina-2000-(bertonatticorcuera).pdf

Fink, W.L., \& Weitzman, S.H. (1974). The so-called cheirodontin fishes of Central America with descriptions of two new species (Pisces: Characidae). Smithsonian Contributions to Zoology 172: 1-46. DOI:10.5479/si.00810282.172

Flores, S.A., Hirt, L.M., \& Araya, P.R. (2015). Estructura y dinámica de la comunidad íctica del Arroyo Yabotí. Reserva de Biosfera Yabotí. Misiones (Argentina). Fish diversity and community structure of Yabotí Stream. Yabotí Biosphere Reserve. Misiones (Argentina). Revista Mexicana de Biodiversidad, 86(2015), 386-395. DOI:10.1016/j.rmb.2015.04.004

Flores, S.A., Araya, P.R., Serrano, M.J., Barboza, A.G., \& Hirt, L.M. (2020). Estructura de la comunidad íctica del arroyo Paraíso afluente del río Uruguay. Misiones. Argentina. Biología Acuática, 34, 1-12. DOI:10.24215/16684869e009

López, H.L., Menni, R.C., Donato, M., \& Miquelarena, A. (2008). Biogeographical revision of Argentina (Andean and Neotropical Regions): An analysis using freshwater fishes. Journal of Biogeography 35, 9, 1564-1579. DOI:10.1111/j.1365-2699.2008.01904.x

Lucena, A.A., Castro, J.B., \& Bertaco, V.A. (2013). Three new species of Astyanax from drainages of southern Brazil (Characiformes: Characidae). Neotropical Ichthyology, 11 (3), 537-552. DOI:10.1590/S1679-62252013000300007

Ministerio de Ecología y Recursos Naturales Renovables Misiones. (2020). https://ecologia.misiones.gob.ar/ anp-sistemas-de-areas-protegidas-naturales /

Mirande, J.M., \& Koerber, S. (2020). Checklist of the freshwater fishes of Argentina (CLOFFAR-2). Ichthyological Contributions of Peces Criollos, 72, 1-81 https:// pecescriollos.de/

Mittermeier, R.A., Myers, N., Thomsen, J.B., da Fonseca, G.A., \& Olivieri, S. (1998). Biodiversity hotspots and major tropical wilderness areas: approaches to setting conservation priorities, Conservation Biology, 12(3), 51620. https://www.deepdyve.com/lp/wiley/biodiversityhotspots-and-major-tropical-wilderness-areasapproaches-ntn9yyQeIs

Myers, N., Mittermeier, R.A., Mittermeier, C.G., da Fonseca, G.A., \& Kent, J. (2000). Biodiversity hotspots for conservation priorities. Nature, 403(6772), 853-8. DOI:10.1038/35002501

Ringuelet, R.A. (1975). Zoogeografía y ecología de los peces de aguas continentales de la Argentina y consideraciones sobre las áreas ictiológicas de América del Sur. Ecosur, 2(3), 1-122. http: //sedici.unlp.edu.ar/bitstream/handle/10915/48003/ Documento_completo__pdf?sequence $=1 \&$ isAllowed $=\mathrm{y}$

Terán, G.E., Alonso, F., García, I., Calviño, P., \& Mirande, J.M. (2016). Occurrence of Astyanax dissensus Lucena \& Thofehrn, 2013 (Teleostei: Characidae) in Argentina. Check List, 12(1), 1828. DOI:10.15560/12.1.1828

Terán, G.E., Benitez, M.F., \& Mirande, J.M. (2020). Opening the Trojan horse: phylogeny of Astyanax, two new genera and resurrection of Psalidodon (Teleostei: Characidae). Zoological Journal of the Linnean Society, 190(4), 12171234. DOI:10.1093/zoolinnean/zlaa019 